\title{
Sub-Leading Effects and the Field Range in Axion Inflation
}

\author{
Ivonne Zavala* \\ Department of Physics, Swansea University, Swansea, SA2 8PP, UK \\ E-mail: e.i.zavalacarrasco@swansea.ac.uk
}

\begin{abstract}
An attractive inflaton candidate is an axion slowly rolling down a flat potential, protected by a perturbative shift symmetry. Realisations of axion inflation within natural and monomial inflation have been recently disfavoured by observations and are difficult to embed in string theory. We show that sub-leading, but significant non-perturbative corrections to the axion potential can superimpose sharp cliffs and gentle plateaus into the potential, whose overall effect is to enhance the number of e-folds of inflation. Sufficient inflation is therefore achieved for smaller field ranges compared to the potential without such corrections and the tensor-to-scalar ratio results un-observably small, but there is a large negative running of the spectral index. This brings both single-field chaotic and natural inflation in UV complete theories like string theory, back into the favoured region of current observations with distinctive signatures. In passing, we derive an upper bound on the tensor-to-scalar ratio $r$, for models of inflation in perturbative string theory.
\end{abstract}

38th International Conference on High Energy Physics 3-10 August 2016

Chicago, USA

${ }^{*}$ Speaker. 


\section{Introduction}

The latest results from Planck and BICEP2/Keck [1] agree with the simplest inflationary scenario where a period of quasi exponential acceleration is driven by a single scalar field, slowly rolling down a very flat potential, whose quantum fluctuations seeded the large scale structures we observe today. One of the biggest challenges in slow-roll inflation is that higher order corrections to the scalar potential, encoded in higher dimensional operators of the form $\mathscr{O}_{d}=c_{d} V(\phi)\left(\phi / M_{P l}\right)^{d-4}$ generically steepen the potential and spoil the slow-roll conditions. Moreover, even for moderately large field values $\phi \gtrsim M_{P l}$, Planck suppressed operators are dangerously large, and can prevent inflation from occurring at all.

Invoking a symmetry is an attractive possibility to prevent large quantum corrections. Such a symmetry is provided, for example, if the inflaton is an axion with a shift symmetry. When the symmetry is broken non-perturbatively to a discrete symmetry, the potential is sufficiently flat for slow-roll, for large values of the symmetry breaking scale, or axion decay constant, $f \gtrsim 4 M_{P l}$, and requires super-Planckian field displacements [7]. When the symmetry is broken, e.g. spontaneously, one has an axionic realisation of monomial inflation, whose attempted string theoretic embeddings are known as axion monodromy [8]. Axion models give vanilla natural or monomial inflation predictions only to leading order. Whilst the effective potential is protected from dangerous perturbative corrections, non-perturbative effects like instantons give further contributions of the form $\sum_{n} \Lambda_{n}^{4} \cos (n \phi / f)$, where $\Lambda_{n}$ are mass scales. These corrections introduce large and frequent modulations into the potential, trapping the inflaton in a local minimum before it reaches the global one, and obstructing large numbers of e-folds of slow-roll natural inflation [9]. On the other hand, when non-perturbative corrections correspond to tiny, frequent superimposed features in the slow-roll potential, their impact on the background trajectory of the inflaton is negligible, whilst leaving only small imprints on the CMB such as a large, possibly oscillating running of the scalar spectral index [10].

Assuming slow-roll from the time the observed perturbations in the CMB exited the horizon up to the end of inflation, the amplitude of the tensor modes, encoded in the tensor-to-scalar ration, $r$, can be related to the inflaton field excursion via the Lyth bound [2] and the inflationary scale, giving

$$
\frac{\Delta \phi}{M_{P l}} \gtrsim 2 \times\left(\frac{r}{0.01}\right)^{1 / 2}, \quad V_{\text {inf }}^{1 / 4} \simeq\left(\frac{r}{0.1}\right)^{1 / 4} \times 1.8 \times 10^{16} \mathrm{GeV} .
$$

Therefore, an observation of primordial gravitational waves with $r \sim 10^{-1}-10^{-2}$ would fix the scale of inflation to be around the GUT scale and the inflaton field range to be super-Planckian, implying that inflation is highly sensitive to quantum gravity effects. UV completions of high scale/large field inflation via string theory are challengins, due to the proximity of the inflationary scale 1.1 and the string scale, which is typically $M_{s} \lesssim 10^{17} \mathrm{GeV}$ for perturbative string theory. This proximity puts the validity of $4 \mathrm{D}$ effective field theory during inflation under pressure, where, in order to be able to neglect massive string excitations and Kaluza-Klein modes, a hierarchy $V_{\text {inf }}^{1 / 4} \lesssim$ $M_{k k} \lesssim M_{s} \lesssim M_{P l}$ is required [4, 6], $M_{k k}$ being the compactification scale.

Here we show that non-perturbative corrections to axion models of inflation not only allow for slow-roll inflation, but can even help it with important implications for the field range, the decay constant and the cosmological predictions. We quote here the relevant measurements and 
bounds on the CMB observables from Planck 2015/BICEP2-Keck [1]. For the scalar perturbations, the Planck analysis gives (including the tensor perturbations at the pivot scale $k_{*}=0.05 \mathrm{Mpc}^{-1}$ and 68\%CL for Planck TT+lowP): $n_{s}=0.9667 \pm 0.0066, \alpha_{s} \equiv d n_{s} / d \ln k=-0.0126_{-0.0087}^{+0.0098}, r<$ 0.07 (at95\%CL).

\section{Sub-leading Corrections in Monomial Inflation}

We start by studying the effects of sub-leading corrections to chaotic (monomial) axion inflation. To do this, we consider the following axionic monomial potential with non-perturbative corrections $[11,12]$

$$
V(\phi)=A+\frac{1}{2} m^{2} \phi^{2}+\lambda \phi \cos \left(\frac{\phi}{f}\right) .
$$

For $\lambda=A=0$, we recover $\phi^{2}$ inflation, which gives a field range of $\Delta \phi \sim 15 M_{P l}$, with a tensor to scalar ratio $r \sim 0.12$ and an inflationary scale of the order of the GUT scale 1.1. While it gives a consistent value for the spectral index $n_{s} \sim 0.966$, this model is basically excluded by the latest results on $r$. We now see how this can change when we take into account subleading modulation effects due to higher order corrections such as non-perturbative terms in string theory constructions.

Let us consider the interesting case $\lambda / f<m^{2}$, taking e.g. $m^{2} / d^{4}=10 M_{P l}^{2}, f=1 / 3 M_{P l}$, and $\lambda / d^{4}=3.3 M_{P l}^{3}$ (where we have scaled the parameters by $d^{2}=9.3 \times 10^{-8}$ to match the normalisation of scale perturbations and for convenience with the numerics, also $t \rightarrow t / d^{2}$ ). We draw the potential in the left picture in Fig. 1, together with the corresponding smooth $\phi^{2}$ model with $\lambda=0$. One can now easily compute the $\mathrm{CMB}$ observables from the slow-roll parameters at horizon crossing for the pivot scale:

$$
n_{s}=1-4 \varepsilon+4 \delta, \quad \alpha_{s}=-8 \xi+20 \varepsilon \delta-8 \varepsilon^{2}, \quad r=16 \varepsilon,
$$

where $\varepsilon \equiv 2 \frac{H^{\prime 2}}{H^{2}}, \delta \equiv \frac{H^{\prime \prime}}{H}, \xi \equiv \frac{H^{\prime \prime \prime} H^{\prime}}{H^{2}}, H$ is the Hubble parameter and a prime denotes derivative with respect to the inflaton. Assuming the pivot scale crossed the horizon at around $N_{e} \sim 55$ e-folds before the end of inflation yield the following values for the inflationary observables

$$
n_{s}=0.9667, \quad r=3.1 \times 10^{-5}, \quad \alpha_{s}=-0.015
$$

in the ballpark of the Planck measurements and constraints given by Planck15. The field range and scale of inflation for the model with sub-leading corrections turn out to be,

$$
\Delta \phi \sim 3.2 M_{P l}, \quad V_{\text {inf }}^{1 / 4}=9.9 \times 10^{-4} M_{P l} .
$$

Thus, non-perturbative corrections can modify a large field monomial model of inflation to one with intermediate field range and inflationary scale, making a consistent perturbative string theory realisation of the model possible. The distinctive signature of such a scenario is a suppressed tensor mode and a large negative running of the spectral index. 

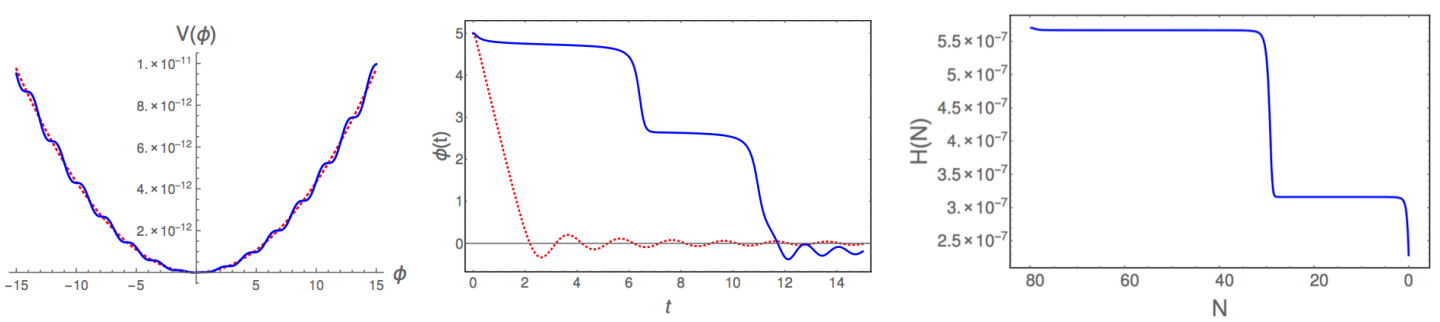

Figure 1: Scalar potential and inflaton's evolution in time for the smooth and bumpy chaotic model and Hubble parameter's evolution as a function of the number of e-folds $N$ for the bumpy chaotic model.

\section{Sub-leading Corrections to Natural Inflation}

We now consider a single field modulated natural potential, which is generically predicted by string theory models:

$$
V(\phi)=A+\Lambda^{4}\left(1+\cos \left(\frac{\phi}{f}\right)\right)+\tilde{\Lambda}^{4}\left(1+\cos \left(\frac{\phi}{\tilde{f}}\right)\right)
$$

where $\tilde{\Lambda}<\Lambda$ and $\tilde{f}<f$ parameterise the bumps. For example, choosing $\Lambda^{4} / d^{4}=1 M_{P l}^{4}, f=1 M_{P l}$ and $\tilde{f}=1 / 3 M_{P l}$, we tune $\tilde{\Lambda}^{4} / d^{4}=0.3329 M_{P l}^{4}$ (where now $d^{2}=9.1 \times 10^{-8}$ ) to ensure the turns in the potential are close to stationary points. The model with sub-leading corrections again has a step-like shape, with steep regions connected by a plateau. The potential and scalar field's evolution for the smooth and bumpy models are given in Fig. 2 as well as the Hubble parameter's evolution as a function of the number of e-folds. Almost all the inflation proceeds in a slow-roll fashion, although there are large fluctuations in the slow-roll parameters when rolling down the steep slopes of the steps. Take the pivot scale to cross the horizon at $N_{e} \sim 54$ e-folds before the end of inflation yield the following values for the cosmological observables:

$$
n_{s}=0.9677, \quad r=3.5 \times 10^{-7}, \quad \alpha_{s}=-0.0025,
$$

in agreement with the Planck measurements. As in the monomial model, tensor modes are undetectably small, whereas there is a large negative running of the spectral index. The field range and scale of inflation in for this case turn out to be

$$
\Delta \phi=1.0 M_{P l}, \quad V_{\text {inf }}^{1 / 4}=3.2 \times 10^{-4} M_{P l} .
$$

We reiterate that non-perturbative corrections have made it possible to achieve single field, natural inflation with a Planckian axion decay constant, $f \sim M_{P l}$, moderate field range and intermediate inflationary energy scale. Thus a consistent embedding in perturbative string theory becomes possible.

\section{Conclusions}

Non-perturbative corrections typically introduce modulations into monomial and natural inflaton potentials. Whereas it was previously assumed that such bumps would either spoil slow-roll 

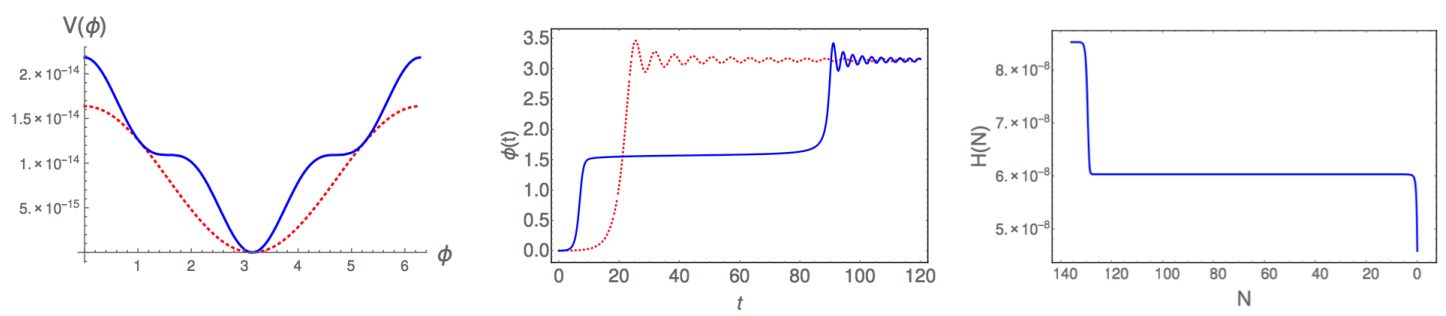

Figure 2: Scalar potential and field evolution for the smooth and bumpy natural models and Hubble's parameter evolution as a function of the number of e-folds for the bumpy model.

inflation [9] or produce negligible corrections to the slow-roll dynamics [8, 10, 11], we have seen that when the modulations take the form a series of steep cliffs and gentle plateaus, the large Hubble friction (or drag) and the sharp reduction in acceleration at the end of the cliffs, cause the inflaton to roll slowly whenever it reaches a plateau. Hence, although slow-roll parameters have large fluctuations through the sharp cliffs, the plateaus provide regions of slow-roll that produce many e-folds of inflation.

Consequently, both single field monomial and natural inflation models can give sufficient efolds for sub-Planckian axion decay constants, moderate field ranges and inflationary scales, when non-perturbative effects are included. This puts them back into the favoured region of current observations and the weakly coupled, supergravity limit of string theory. Such a scenario has distinctive signatures. In particular, the benchmark models considered here predict tensor modes 24 orders of magnitude below the projected bounds of future observations like LiteBIRD, $r \lesssim 10^{-3}$. Moreover, bumpy models also predict large, negative values for the running of the scalar spectral index over the scales probed by the $\operatorname{CMB}\left(\alpha_{s} \approx-10^{-2},-10^{-3}\right.$, respectively), and small running of the running $\left(\beta_{s} \approx-10^{-4},-10^{-5}\right.$, respectively).

We considered simple potentials within effective field theory, and it would be important to understand whether the potentials and parameters emerging from string compactifications can fullfill the requisite tunings, such as for example in the models discussed in [12]. Also, a more detailed study of the inflationary observables would be very interesting, for example consequences of the running spectral index on all the scales probed by the $\mathrm{CMB}$, and implications of the bumps for non-Gaussianities.

Acknowledgments. I am thankful to my collaborators on the topics discussed here, K. Kooner, N. Cabo-Bizet, O. Loaiza-Brito, S. Parameswaran and G. Tasinato.

\section{References}

[1] P. A. R. Ade et al. [Planck Collaboration], "Planck 2015 results. XX. Constraints on inflation," Astron. Astrophys. 594 (2016) A20 doi:10.1051/0004-6361/201525898 [arXiv: 1502.02114 [astro-ph. CO] ]; P. A. R. Ade et al. [BICEP2 and Keck Array Collaborations], "Improved Constraints on Cosmology and Foregrounds from BICEP2 and Keck Array Cosmic Microwave Background Data with Inclusion 
of 95 GHz Band," Phys. Rev. Lett. 116 (2016) 031302 doi:10.1103/PhysRevLett.116.031302 [arXiv:1510.09217 [astro-ph.co]].

[2] D. H. Lyth, "What would we learn by detecting a gravitational wave signal in the cosmic microwave background anisotropy?,” Phys. Rev. Lett. 78 (1997) 1861 doi:10.1103/PhysRevLett.78.1861 [hep-ph/9606387].

L. Boubekeur and D. H. Lyth, "Hilltop inflation," JCAP 0507 (2005) 010 doi:10.1088/1475-7516/2005/07/010 [hep-ph/0502047];

J. Garcia-Bellido, D. Roest, M. Scalisi and I. Zavala, "Lyth bound of inflation with a tilt," Phys. Rev. D 90 (2014) no.12, 123539 doi:10.1103/PhysRevD.90.123539 [arXiv:1408.6839 [hep-th] ].

[3] N. Arkani-Hamed, L. Motl, A. Nicolis and C. Vafa, "The String landscape, black holes and gravity as the weakest force," JHEP 0706 (2007) 060 doi:10.1088/1126-6708/2007/06/060 [hep-th/0601001].

[4] K. Kooner, S. Parameswaran and I. Zavala, "Warping the Weak Gravity Conjecture," Phys. Lett. B 759 (2016) 402 doi:10.1016/j.physletb.2016.05.082 [arXiv:1509.07049 [hep-th] ].

[5] S. Parameswaran, G. Tasinato and I. Zavala, "Subleading Effects and the Field Range in Axion Inflation," JCAP 1604 (2016) no.04, 008 doi:10.1088/1475-7516/2016/04/008 [arXiv:1602.02812 [astro-ph.CO]].

[6] S. L. Parameswaran and I. Zavala, "Prospects for Primordial Gravitational Waves in String Inflation," Int. J. Mod. Phys. D 25, 1644011 (2016) doi:10.1142/S0218271816440119 [arXiv:1606. 02537 [hep-th]].

[7] K. Freese, J. A. Frieman and A. V. Olinto, "Natural inflation with pseudo - Nambu-Goldstone bosons,” Phys. Rev. Lett. 65 (1990) 3233. doi:10.1103/PhysRevLett.65.3233

[8] E. Silverstein and A. Westphal, "Monodromy in the CMB: Gravity Waves and String Inflation," Phys. Rev. D 78 (2008) 106003 doi:10.1103/PhysRevD.78.106003 [arXiv: 0803.3085 [hep-th] ]; L. McAllister, E. Silverstein and A. Westphal, "Gravity Waves and Linear Inflation from Axion Monodromy,” Phys. Rev. D 82 (2010) 046003 doi:10.1103/PhysRevD.82.046003 [arXiv:0808.0706 [hep-th]]

[9] T. Banks, M. Dine, P. J. Fox and E. Gorbatov, "On the possibility of large axion decay constants," JCAP 0306 (2003) 001 doi:10.1088/1475-7516/2003/06/001 [hep-th/ 0303252]

[10] T. Kobayashi and F. Takahashi, "Running Spectral Index from Inflation with Modulations," JCAP 1101 (2011) 026 doi:10.1088/1475-7516/2011/01/026 [arXiv:1011.3988 [astro-ph.co]]

[11] R. Flauger, L. McAllister, E. Silverstein and A. Westphal, "Drifting Oscillations in Axion Monodromy," arXiv:1412.1814 [hep-th];

T. Kobayashi, A. Oikawa and H. Otsuka, "New potentials for string axion inflation," Phys. Rev. D 93 (2016) no.8, 083508 doi:10.1103/PhysRevD.93.083508 [arXiv:1510.08768 [hep-ph]].

[12] N. Cabo Bizet, O. Loaiza-Brito and I. Zavala, "Mirror quintic vacua: hierarchies and inflation,” JHEP 1610 (2016) 082 doi:10.1007/JHEP10(2016)082 [arXiv:1605.03974 [hep-th]]. 